
JNM
J Neurogastroenterol Motil, Vol. 22 No. 3 July, 2016
pISSN: 2093-0879 elSSN: 2093-0887
http://dx.doi.org/10.5056/jnm15166

\title{
Esophageal Submucosal Injection of Capsaicin but Not Acid Induces Symptoms in Normal Subjects
}

\author{
Robert H Lee, ${ }^{1}$ Hariprasad Korsapati, ${ }^{2,3}$ Vikas Bhalla, ${ }^{2,3}$ Nissi Varki, ${ }^{4}$ and Ravinder K Mittal ${ }^{2,3 *}$ \\ ${ }^{I}$ Division of Gastroenterology, University of California Irvine, Irvine, CA, USA; ${ }^{2}$ Division of Gastroenterology, University of California, San \\ Diego, San Diego, CA, USA; ${ }^{3}$ Gastroenterology Section, San Diego Veterans Administration Medical Center, La Jolla, CA, USA; and ${ }^{4}$ Department \\ of Pathology, University of California San Diego, San Diego, CA, USA
}

\section{Background/Aims}

Transient receptor potential vanilloid-1 (TRPV1) is a candidate for mediating acid-induced symptoms in the esophagus. We conducted studies to determine if the presence of acid in the mucosa/submucosa and direct activation of TRPV1 by capsaicin elicited symptoms in normal healthy subjects. We also studied the presence of TRPV1 receptors in the esophagus.

\section{Methods}

Unsedated endoscopy was performed on healthy subjects with no symptoms. Using a sclerotherapy needle, normal saline (pH 2.07.5) was injected into the mucosa/submucosa, $5 \mathrm{~cm}$ above the $Z$ line. In a separate group of healthy subjects, injection of capsaicin and vehicle was also studied. Quality of symptoms was reported using the McGill Pain Questionnaire, and symptom intensity using the visual analogue scale (VAS). Immunohistochemistry was performed on 8 surgical esophagus specimens using TRPV1 antibody.

\section{Results}

Acid injection either did not elicit or elicited mild symptoms in subjects at all pH solutions. Capsaicin but not the vehicle elicited severe heartburn/chest pain in all subjects. Mean VAS for capsaicin was $91 \pm 3$ and symptoms lasted for $25 \pm 1$ minutes. Immunohistochemistry revealed a linear TRPV1 staining pattern between the epithelial layer and the submucosa that extended into the papillae. Eighty-five percent of papillae stained positive for TRPV1 with a mean 1.1 positive papillae per high-powered field.

\section{Conclusions}

The mechanism of acid-induced heartburn and chest pain is not the simple interaction of hydrogen ions with afferents located in the esophageal mucosa and submucosa. TRPV1 receptors are present in the lamina propria and their activation induces heartburn and chest pain.

(J Neurogastroenterol Motil 2016;22:436-443)

\section{Key Words}

Capsaicin; Chest pain; Esophagus; Heartburn; TRPV1 receptor

Received: October 13, 2015 Revised: January 14, 2016 Accepted: January 27, 2016

(a) This is an Open Access article distributed under the terms of the Creative Commons Attribution Non-Commercial License (http://creativecommons. org/licenses/by-nc/4.0) which permits unrestricted non-commercial use, distribution, and reproduction in any medium, provided the original work is properly cited.

*Correspondence: Ravinder K Mittal, MD

Division of Gastroenterology, University of California, 3350 La Jolla Village Drive 111D, San Diego, CA 92161, USA

Tel: +1-858-552-7556, Fax: +1-858-552-4327, E-mail: rmittal@ucsd.edu 


\section{Introduction}

Heartburn and "angina like chest pain" originating from the esophagus are common symptoms encountered in clinical practice. Acid reflux, esophageal dysmotility and hypersensitivity of the esophagus are thought to be pathophysiological mechanisms underlying these symptoms. The molecular basis of esophageal nociception is an area of intense interest, both from the vantage point of basic knowledge as well formulating possible new therapeutic strategies.

How does acid reflux cause heartburn and chest pain? It is suggested that acid-induced damage to the esophageal epithelium allows acid/proton ions to permeate into the mucosa/submucosa where it activates acid sensitive nerves to induce heartburn/chest pain. ${ }^{1}$ A large body of literature in animal and humans experiments shows that acid in the esophagus results in dilated intercellular space. ${ }^{2,3}$ Furthermore, patients with heartburn symptoms and a normal appearing mucosa on endoscopy have dilated intercellular spaces that could allow acid/proton ions to permeate into the mucosa/submucosa. Studies also show that spinal nerve endings are located in the submucosa/mucosa of the esophagus and that they are possibly the transduction site of acid-induced sensation from the esophagus. ${ }^{4}$ Along those lines, acid perfusion into the esophageal lumen of normal healthy subjects does not cause symptoms (a negative Bernstein test) because normal subjects have an intact epithelium. On the other hand, acid perfusion into the esophagus in patients with reflux disease causes symptoms (a positive Bernstein test $)^{5}$ because the presence of dilated intercellular spaces allows access to acid-sensitive nerve endings located in the submucosa. If the above hypotheses were to be correct, injection of acid directly into the submucosa of normal healthy subjects should induce symptoms, an experiment that has never been done.

Basic science and clinical data in humans suggests that the vanilloid receptors are involved in somatic as well as visceral nociception. Alkaloid resembling vanillyl (vanilloids) are the natural ingredients in chili powder or capsicum. Vanilloids act through transient receptor potential vanilloid receptors (TRPV) to activate action potentials in the afferent nerve fibers that carry pain sensation to the central nervous system. ${ }^{6}$ Even though multiple vanilloid receptors (TRPV1-6) have been identified, TRPV1 is thought to be most likely involved in nociception. ${ }^{7,8}$ TRPV1 activity has been found in the esophageal mucosa as well as in several other areas of the gastrointestinal tract in humans., ${ }^{9,10}$ Capsaicin, the active ingredient in chili powder, is an agonist that is specific for TRPV1.
Heat and protons also activate TRPV1 receptors. Oral ingestion and infusion of a chili powder solution is known to elicit heartburn symptoms in normal healthy subjects. ${ }^{11,12}$

Therefore, the goals of our study were to determine: (1) if esophageal mucosal/submucosal injection of acid and capsaicin elicits symptoms in normal healthy subjects. We were interested in the nature and location of symptoms elicited by localized proton/ vanilloid receptor activation, ie, if these symptoms have resemblance to symptoms experienced by patients suffering from esophageal disorders, and (2) the presence of TRPV1 receptors in full thickness specimens of the human esophagus.

\section{Materials and Methods}

The Institutional Review Board of the University of California San Diego approved the study protocol, and all subjects signed an informed consent prior to enrollment in the study. Before the start of study, subjects were familiarized with the McGill Pain Questionnaire and symptom/sensation terminology. They were informed that heartburn implied a burning sensation between the xiphoid process and the manubrium sternum, and that chest pain was a pressure/ squeeze sensation. Subjects were also asked to describe in their own words the description of the sensation experienced in response to injection.

Upper endoscopy was performed using a $4.9 \mathrm{~mm}$ diameter endoscope (GIF-XP160, Olympus, Tokyo, Japan). The nasal and pharyngeal mucosa was anesthetized using a $2 \%$ lidocaine solution and viscous lidocaine gel. Subjects were not given any sedating medication for the endoscopy. Using a 23 gauge, $4 \mathrm{~mm}$ long sclerotherapy needle (Boston Scientific, Natick, MA, USA), acidified saline or capsaicin was injected in the mucosa/submucosa of the esophagus.

\section{Symptoms Following Injection of Capsaicin}

This cross-over study design involved nine healthy male volunteers, age 21-50 years with no history of heartburn or gastroesophageal reflux disease symptoms. All subjects underwent unsedated trans-nasal upper endoscopy with a single esophageal intubation, and underwent serial injections of test solutions using a sclerotherapy needle. One hundred micrograms of capsaicin dissolved in 0.1 $\mathrm{mL}$ of vehicle (20\% 2 hydroxypropyl-beta-cyclodextrin solution; Sigma-Aldrich, St. Louis, MO, USA) and vehicle were injected underneath the esophageal submucosa in a single blinded fashion (subjects but not the investigator were blinded). The selected capsaicin dose was based on prior studies that utilized intradermal 
capsaicin injection. ${ }^{13,14}$ Single injections of $0.1 \mathrm{~mL}$ of vehicle and capsaicin (vehicle always first) were made $5 \mathrm{~cm}$ above the $Z$ line. Following injections, subjects reported the location, severity and quality of symptoms, every 2 minutes for 30 minutes using the McGill Pain Questionnaire. ${ }^{15}$ The symptom severity was scored using a $100 \mathrm{~mm}$ visual analog scale (VAS). A 30 minute time period was allowed in between the 2 injections.

\section{Symptoms Following Injection of Acid}

Eight healthy asymptomatic volunteers, age $21-50$ years (6 males) participated in this part of the study. These subjects were different from the ones that participated in the capsaicin study. Isoosmolar normal saline of $\mathrm{pH}$ ranging from 2-7.5 was prepared under sterile conditions. Solution of acidified normal saline $(0.1-$ $1.0 \mathrm{~mL}$ ) was injected in the mucosa/submucosa of the esophagus in a double-blinded fashion (8-10 injections per subject) (neither investigator nor subject knew the $\mathrm{pH}$ of injected solution). Subjects scored the symptom location and quality using McGill Pain Questionnaire, every 2 minutes until symptoms disappeared. A 10-minute period was allowed between each injection. The symptom severity was scored using VAS. Injections were made mostly at $5 \mathrm{~cm}$ and some at $10 \mathrm{~cm}$ above the $\mathrm{Z}$ line.

\section{Expression of Transient Receptor Potential Vanilloid-1 in the Esophagus}

Surgical specimens from 8 patients who had undergone esophagectomy for adenocarcinoma of the esophagus were studied. Specimens were obtained flash frozen from the Cooperative Human Tissue Network (CHTN) in accordance with National Cancer Institute guidelines. All histological sections used were from areas that had no histological evidence of cancer. Ten micron frozen sections were overlaid with pre-determined concentrations of rabbit anti-TRPV1 antibody (Affinity Bioreagents, Golden, CO, USA). Negative controls included sections with no primary, or sections that received normal rabbit serum at the same concentration that was used as the TRPV1 antibody, and sections that were overlaid with an inhibitory peptide (Affinity Bioreagents) along with the antiTRPV1 antibody. One specimen was co-stained with monoclonal rabbit anti-neurofilament antibody (Signet Laboratories, Dedham, MA, USA), and some slides were double immunostained with anti-TRPV1 and anti-neurofilament antibodies. The investigators reviewed all slides with an expert pathologist (N.V.).

A total of 10 high power fields (HPF) of the lamina propria, longitudinal muscle and circular muscle layers were also examined under $\times 40$ for TRPV1 staining. The number of papillae staining positive for TRPV1 per HPF was calculated, as was the percentage of total papillae staining positive for TRPV1. The intensity of TRPV1 staining in the papillae was graded based upon the following scale: strong, greater than $2 / 3$ of the height of the papillae was stained; moderate, 1/3-2/3 papilla was stained; and weak, less than $1 / 3$ of the papillae stained.

\section{Statistical Methods}

The duration and peak intensity of symptoms following the vehicle and capsaicin injections were determined from the questionnaire. A paired $t$ test was used to determine the difference in symptoms between the 2 types of injections for capsaicin and vehicle. All data are presented as mean $\pm \mathrm{SD}$.

\section{Results}

\section{Symptom Response to Capsaicin}

The esophageal mucosa, as seen on endoscopy was normal in all subjects. In response to vehicle injection, either no symptoms or mild symptoms were reported. When symptoms were reported after vehicle injection, they lasted for less than 2 minutes. Figure 1 shows the quantitative result of heartburn felt by one subject following injection of placebo and capsaicin. Placebo injection resulted in heartburn sensation that was graded 18 on the VAS and the duration of sensation was less than 2 minutes. On the other hand, following

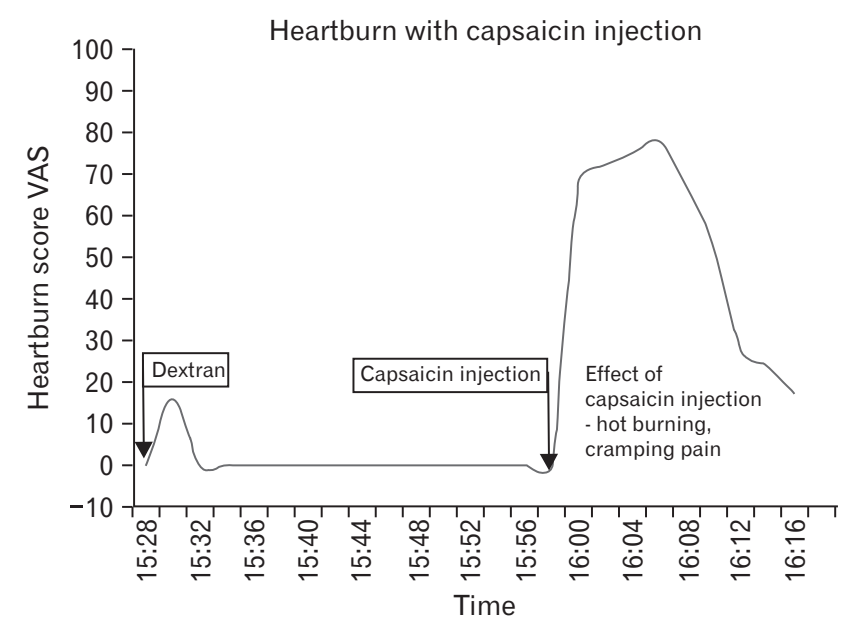

Figure 1. Heartburn scores for one subject as measured on a $100 \mathrm{~mm}$ visual analogue scale (VAS) with injection of placebo (dextran) and capsaicin. Capsaicin injection resulted in a 4-fold increase in pain scores as compared to placebo and longer duration of symptoms as compared to placebo. 
capsaicin injection, the peak intensity of heartburn was 80 with a mean duration $>25$ minutes. The mean heartburn severity after capsaicin injection in all subjects was $91 \pm 3$ and the mean duration was $25 \pm 1$ minutes. In contrast, the mean severity and duration of symptoms following placebo injection were $27 \pm 2$ and $1 \pm 1$ minute, respectively ( $P<0.001$ for both compared with capsaicin).

The nature and severity of sensation following capsaicin injection is shown in the Table. All subjects experienced heartburn, and $8 / 9$ reported chest pressure/cramp-like sensations. Six subjects described their pain as an aching type of sensation. Interestingly, 3 patients reported spasm like pain radiating to the back, ie, in the interscapular and lumbar regions. Two subjects experienced nausea and vomiting. Seven subjects described their symptoms as severe and 2 subjects graded them as moderate.

\section{Symptom Response to Acidified Saline}

The esophageal mucosa, as seen on endoscopy, was normal in all subjects. Four of the eight subjects reported either no symptoms or barely perceptible symptoms following injection of acidic solution. The other 4 subjects reported symptoms $>50$ on the VAS to one or more $\mathrm{pH}$ solution injections, however there was no dose response observed in any of the subjects, eg, one subject reported symptom scores of $26,38,49,9,39,16$, and 49 at $\mathrm{pH}$ of $4.0,4.5,5.0$, $6.0,6.5,7.0$, and 7.5 respectively. Median pain scores experienced

Table. Summary of Pain Descriptors Described by Subjects in Response to Capsaicin as Defined on the McGill Pain Questionnaire

\begin{tabular}{|c|c|c|c|c|c|c|c|c|}
\hline & $\begin{array}{c}\text { Shooting } \\
\text { pain }\end{array}$ & $\begin{array}{l}\text { Sharp } \\
\text { pain }\end{array}$ & $\begin{array}{c}\text { Cramping } \\
\text { pressure } \\
\text { squeeze }\end{array}$ & $\begin{array}{c}\text { Gnawing } \\
\text { pain }\end{array}$ & $\begin{array}{c}\text { Hot } \\
\text { burning }\end{array}$ & Aching & Sickening & $\begin{array}{c}\text { Back } \\
\text { spasms }\end{array}$ \\
\hline Subject 1 & & & +++ & & +++ & & & \\
\hline Subject 2 & & & +++ & & +++ & +++ & & ++ \\
\hline Subject 3 & & & +++ & +++ & +++ & ++ & & ++ \\
\hline Subject 4 & +++ & +++ & +++ & & +++ & ++ & ++ & +++ \\
\hline Subject 5 & & & +++ & & +++ & & & \\
\hline Subject 6 & & & ++ & & +++ & & +++ & \\
\hline Subject 7 & & & & & +++ & & & \\
\hline Subject 8 & & & +++ & & ++ & +++ & & \\
\hline Subject 9 & & & +++ & & ++ & +++ & & \\
\hline
\end{tabular}

Hot-burning characteristic of pain was a consistent finding in all study subjects.

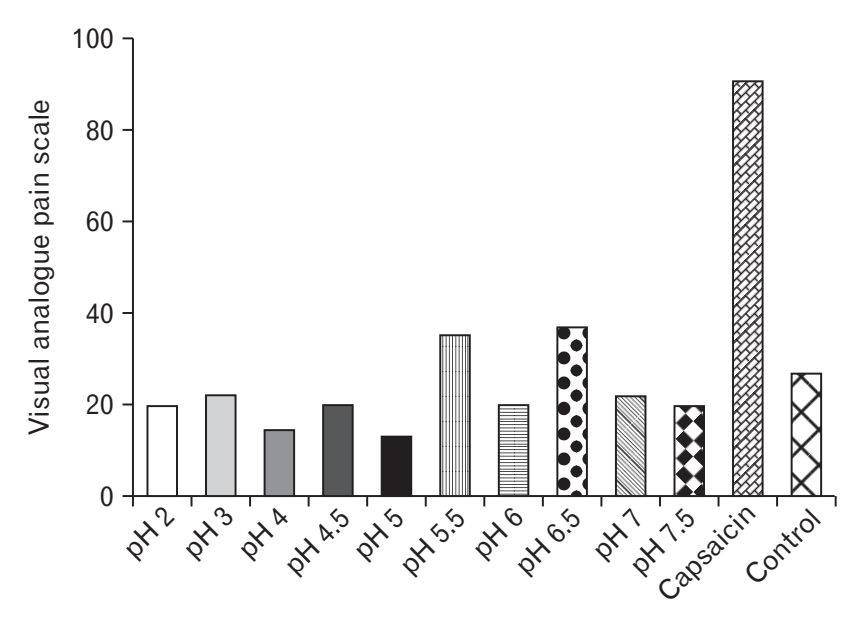

Figure 2. Symptom response to injection of acid and capsaicin into the esophageal submucosa: note that symptoms induced by injection were mild and not increased with the decrease in $\mathrm{pH}$. On the other hand, capsaicin induced severe symptoms. The bars represent the median pain score after injection of acid $(n=8)$ and capsaicin $(n=9)$.

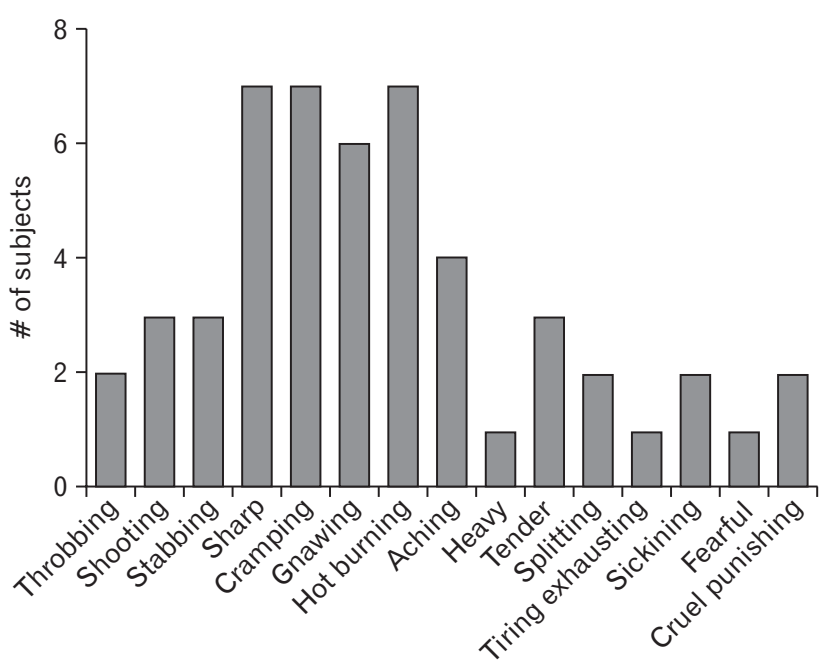

Figure 3. Descriptors of pain reported by subjects receiving mucosal/ submucosal injection of $\mathrm{pH}$ solutions 4.0-7.5. The intensity of pain response using the visual analogue scale was not reproducible at any given $\mathrm{pH}$. 
by subjects receiving the $\mathrm{pH}$ injection, capsaicin and the vehicle are summarized in Figure 2. Overall, there was no relationship between the $\mathrm{pH}$ of the injected solution and severity of symptom score in contrast to the relationship seen with capsaicin injection. In 3 subjects, $\mathrm{pH} 5$ injections were repeated twice and there were no differences in pain scores during the 2 attempts. In another 3 subjects, solutions of two different osmolarities were injected, iso-osmolar (280 mosmolar) and hyperosmolar (330 mosmolar) at $\mathrm{pH}$ 7.5. The symptoms were mild, 20 and 2 (subject 1), 25 and 20 (subject 2), and 5 and 2 (subject 3). In other words, increasing osmolality of the solution did not increase the symptom severity. The characteristics of pain experienced by subjects upon injection of $\mathrm{pH}$ solutions are summarized in Figure 3, with sharp, cramping, gnawing, and burning being the most common descriptors.

\section{Expression of Transient Receptor Potential Vanilloid-1 in the Esophagus}

Immunohistochemical staining (using the TRPV1 specific antibody) was distributed in a linear staining pattern traversing along the junction between the epithelial layer and lamina propria (Fig. 4A). In 7 out of 8 specimens, a linear staining pattern extended from the lamina propria along the basement membrane into the epithelial papillae. Immunostaining was absent in the specimens that were incubated with both the TRPV1 antibodies and the homologous blocking peptide, thus proving the specificity of the immunostaining for TRPV1 (Fig. 4B). Quantitative analysis demonstrated that $445 / 514(87 \%)$ of all epithelial papillae stained positive for TRPV1. A mean of 1.14 TRPV1 positive papillae were seen per HPF. Of papillae staining positive for TRPV 1, 42\% demonstrated
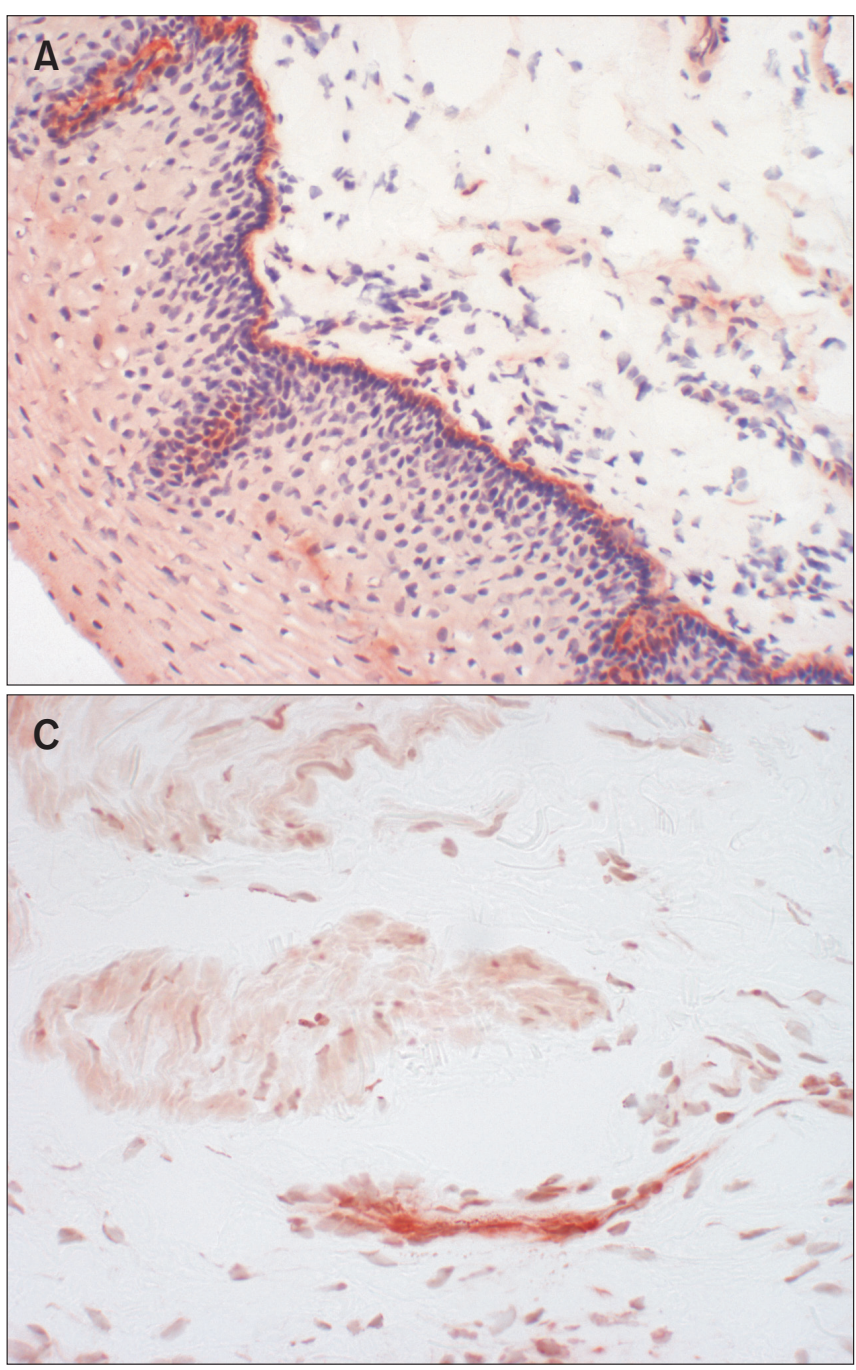

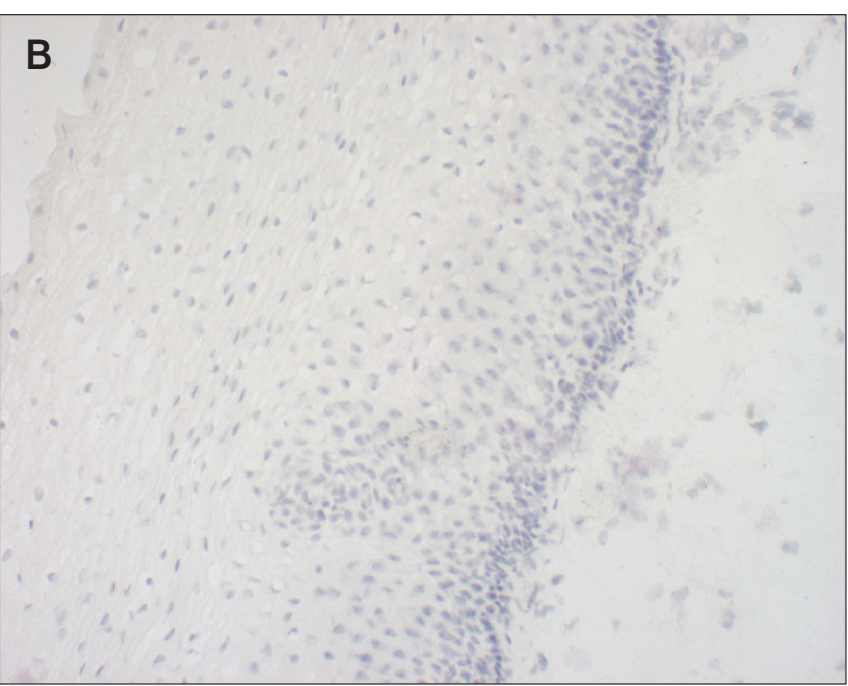

Figure 4. Transient receptor potential vanilloid-1 (TRPV1) Staining. (A) TRPV1 positive structures are seen in a linear pattern extending from the lamina propria and into the epithelial papillae. Upon review of all specimens, $87 \%$ of all papillae were found to exhibit this staining pattern. (B) Staining of specimens using TRPV1 homologous blocking peptide demonstrates complete suppression of prior TRPV1 antibody positive staining structures, thus proving the specificity of the TRPV1 antibody. (C) Specimen stained with the TRPV1 antibody. TRPV1 staining structures are also seen as twig-like bundles in the lamina propria. 
strong staining, $21 \%$ moderate, and $37 \%$ stained weakly.

One specimen that was stained with both the anti-TRPV1 antibody and anti-neurofilament antibody demonstrated TRPV1 activity that appeared as twig-like bundles located in the lamina propria near the circular muscle layer. These twig-like bundles had the morphology of a nerve structure, which was confirmed by the positive immunoreactivity with the anti-neurofilament antibody (Fig. $4 \mathrm{C})$.

\section{Discussion}

In summary, our data shows that acid injection into the esophageal submucosa of healthy asymptomatic subjects produced either mild or no symptoms, and that these symptoms were neither reproducible nor dose dependent. On the other hand, capsaicin injection induced typical symptoms that resemble description of spontaneous heartburn and chest pain reported by patients with reflux disease and esophageal motility disorders. Some subjects even reported radiation of pain into the interscapular region that is known to be associated with the pain originating from the esophagus. Capsaicin also induced some symptoms that may be considered atypical for the esophagus, such as nausea, vomiting, and radiation of pain into the lumbar region. In the majority of subjects, symptoms were quite intense and lasted 25-30 minutes. We also observed TRPV1 immunostaining pattern in the esophageal mucosa that is consistent with the findings of others. ${ }^{16,17}$ Ours is the first study of injection of acid and capsaicin into the mucosa/submucosa of the human esophagus, and raises questions regarding the prevailing hypothesis of how acid reflux causes esophageal pain and heartburn.

The current hypothesis regarding acid-induced esophageal pain is that intra-luminal acid along with pepsin causes damage to the epithelial lining of the esophagus, which may manifest as one of the following phenotypes: (1) erosive esophagitis on endoscopy, (2) normal endoscopy but positive histological changes of esophagitis, ie, increase in inflammatory cells in the mucosa and submucosa, and (3) normal endoscopy and histology but dilated intercellular spaces that are only observed on electron microscopy. ${ }^{18}$ Due to the presence of these dilated intercellular spaces, acid can permeate into the layers of mucosa and submucosa. In fact, a large body of work shows that transmucosal potential difference and baseline electrical impedance of esophageal mucosa (markers of mucosa integrity) is lower in patients with symptoms of heartburn (erosive disease and non-erosive reflux disease), as compared to normal subjects. ${ }^{19}$ Once in the mucosa and submucosa, proton $/ \mathrm{H}^{+}$comes in contact with nociceptive nerve endings to induce heartburn and chest pain. If the above hypothesis were to be accurate, injection of hydrogen ions directly into the mucosa/submucosa should have caused heartburn/ chest pain, which was not observed in our study.

Acid sensitive ion channels (ASIC) and TRPV1 receptors are potential molecular candidates of nociception. Acid at higher $\mathrm{pHs}$ (5-7) activates ASIC receptors and at lower $\mathrm{pH}(<5.0)$ stimulates TRPV1 receptors. ${ }^{20}$ The latter is a multimodal receptor; it can be activated by protons, capsaicin, and heat. We are extremely surprised to find that while capsaicin injection induced severe symptoms, acid injection even at $\mathrm{pH} 2$ did not elicit significant symptoms. At the beginning of our study, our plan was to inject solution of pH 5-7 only, but when we did not observe any significant response we extended our injection protocol to $\mathrm{pH} 2$, but still could not elicit symptoms. We initially started with injection volume of $0.1 \mathrm{~mL}$, similar to capsaicin but then increased to $1 \mathrm{~mL}$ and still found no response. We tried injections at $5 \mathrm{~cm}$ and $10 \mathrm{~cm}$ above the $\mathrm{Z}$ line, as well as different osmolar concentrations but did not induce any symptoms. It is possible that unlike capsaicin, acidic saline either diffuses or is buffered to neutral $\mathrm{pH}$ very quickly in the tissue and is unable to stimulate the desired receptors. However, response to capsaicin was instantaneous, and if rapid diffusion was the issue we should have observed immediate symptoms with rapid disappearance of symptoms, rather than no symptoms, which was not the case. A number of investigators have observed that spontaneous reflux episodes that extend into the proximal esophagus are more likely to be associated with symptoms than those restricted to the distal esophagus, ${ }^{18,21,22}$ which raises the possibility that symptom generation occurs when a relatively large surface of mucosa is exposed to acid and therefore a large number of receptor are stimulated, greater than what was achieved by our localized injection.

Another possibility is that ASIC and TRPV1 are not expressed in significant amounts in normal esophageal mucosa, and it is only the repeated exposure to acid that occurs with chronic reflux disease that leads to up-regulation of ASIC and TRPV1 receptors (one of the mechanisms of acid-induced esophageal hypersensitivity). However, dramatic symptom response following capsaicin injection suggests that TRPV1 receptors are indeed present and functional in normal esophageal mucosa and submucosa. Drewes et $\mathrm{al}^{23}$ demonstrated that capsaicin administration in the ileum (through a stoma) can elicit "burning" and "boring" symptoms. Furthermore, in their studies TRPV1 activation was also found to play a role in the distension-induced visceral hyperalgesia. ${ }^{23,24}$

Capsaicin is a specific agonist of TRPV1 and has been extensively used to study the mechanism of somatic pain (intradermal junction). ${ }^{13,14}$ While the strength of a human experiment, like ours, 
is that it allows qualitative and quantitative assessment of pain, it does not allow detailed study of the molecular mechanism. For example, we could not assess if the mechanism of action of capsaicin in our experiment is specifically through TRPV 1 receptors because there is no TRPV1 antagonist available for human use. We did intend to determine if the acid induced pain is mediated through the ASIC receptors, as was studied by Ugawa et $\mathrm{al}^{20}$ in their studies of somatic pain (dermal injection), by using amiloride, an ASIC receptor antagonist. We actually obtained human IRB and federal IND approval to use amiloride for our studies. However, acid injection did not induce symptoms in a reproducible manner; therefore using amiloride was a moot-point for our experiment. It is interesting that a recent study by Bulsiewicz et $\mathrm{al}^{25}$ found that perfusion of the esophagus with amiloride did not prevent heartburn in patients with a positive Bernstein test, suggesting that the mechanism of acidinduced sensation is not as simple as previously thought.

We found TRPV1 receptors in the majority of the epithelial papillae $(87 \%)$ in the human esophagus. TRPV1 positive structures were found lining the junction between the lamina propria and epithelial layer. To the best of our knowledge, only 2 other studies have looked for the presence of TRPV1 in the human esophagus. Matthews et $\mathrm{al}^{17}$ found that patients with erosive esophagitis had an increased number of TRPV1 positive papillae as compared to normal controls (30\% versus $11.8 \%$ of total papillae). Bhat and Bielefeldt ${ }^{16}$ noticed TRPV1 positivity in $23 \%$ of the papillae. They also found a correlation between the density of TRPV1 innervation and total acid exposure on 24-hour ambulatory $\mathrm{pH}$ monitoring $(P=0.03){ }^{12}$ Bhat et $\mathrm{al}^{16}$ described TRPV1 staining structures that appeared as free nerve endings in the epithelial layer, close to the surface of the mucosa, which we did not observe. Clearly, our immunostaining technique is specific for TRPV1 receptors because no staining was seen in the presence of the TRPV 1 antibody blocking peptide. The higher percentage of total papillae staining positive for TRPV1 $(87 \%)$ found in our study may be because we used whole surgical specimens rather than smaller 1-2 mm endoscopic biopsies used in the other studies. Furthermore, since our specimens were from patients with esophageal adenocarcinoma who likely had significant reflux disease, it is possible that there was up-regulation of TRPV1 receptors.

We do not intend to imply that acid does not cause heartburn/ chest pain. Our study only raises questions about the current hypothesis of how acid induces esophageal symptoms. Furthermore, it casts some doubt on whether acid is sufficient to activate TRPV1 receptors to induce symptoms. Further studies are needed to define the precise site and mechanism of action of acid for induction of esophageal symptoms of heartburn and chest pain.

Financial support: This work was supported by NIH grant RO-1 DK60733.

\section{Conflicts of interest: None.}

Author contributions: Robert H Lee, Hariprasad Korsapati, and Vikas Bhalla all contributed to data collection and data analysis; Nissi Varki interpreted all histopathology and immunostaining; and Ravinder K Mittal contributed to study design, data collection, and data analysis.

\section{References}

1. Barlow WJ, Orlando RC. The pathogenesis of heartburn in nonerosive reflux disease: a unifying hypothesis. Gastroenterology 2005;128:771778.

2. Tobey NA, Carson JL, Alkiek RA, Orlando RC. Dilated intercellular spaces: a morphological feature of acid reflux--damaged human esophageal epithelium. Gastroenterology 1996;111:1200-1205.

3. Woodland P, Sifrim D. Oesophageal mucosal barrier: a key factor in the pathophysiology of non-erosive reflux disease (NERD) and a potential target for treatment. Gut 2014;63:705-706.

4. Rodrigo J, Hernández J, Vidal MA, Pedrosa JA. Vegetative innervation of the esophagus. II. Intraganglionic laminar endings. Acta Anat (Basel) 1975;92:79-100

5. Bernstein LM, Baker LA. A clinical test for esophagitis. Gastroenterology 1958;34:760-781.

6. Caterina MJ, Schumacher MA, Tominaga M, Rosen TA, Levine JD, Julius D. The capsaicin receptor: a heat-activated ion channel in the pain pathway. Nature 1997;389:816-824.

7. Caterina MJ. Transient receptor potential ion channels as participants in thermosensation and thermoregulation. Am J Physiol Regul Integr Comp Physiol 2007;292:R64-R76.

8. Numazaki M, Tominaga M. Nociception and TRP Channels. Curr Drug Targets CNS Neurol Disord 2004;3:479-485.

9. Ward SM, Bayguinov J, Won KJ, Grundy D, Berthoud HR. Distribution of the vanilloid receptor (VR1) in the gastrointestinal tract. J Comp Neurol 2003;465:121-135.

10. Patterson LM, Zheng H, Ward SM, Berthoud HR. Vanilloid receptor (VR1) expression in vagal afferent neurons innervating the gastrointestinal tract. Cell Tissue Res 2003;311:277-287.

11. Milke P, Diaz A, Valdovinos MA, Moran S. Gastroesophageal reflux in healthy subjects induced by two different species of chilli (Capsicum annum). Dig Dis 2006;24:184-188.

12. Rodriguez-Stanley S, Collings KL, Robinson M, Owen W, Miner PB Jr. The effects of capsaicin on reflux, gastric emptying and dyspepsia. Aliment Pharmacol Ther 2000;14:129-134.

13. Hughes A, Macleod A, Growcott J, Thomas I. Assessment of the repro- 
ducibility of intradermal administration of capsaicin as a model for inducing human pain. Pain 2002;99:323-331.

14. Geber C, Fondel R, Krämer HH, Rolke R, Treede RD, Sommer C, Birklein F. Psychophysics, flare, and neurosecretory function in human pain models: capsaicin versus electrically evoked pain. J Pain 2007;8:503514.

15. Kremer E, Atkinson JH Jr. Pain measurement: construct validity of the affective dimension of the McGill Pain Questionnaire with chronic benign pain patients. Pain 1981;11:93-100.

16. Bhat YM, Bielefeldt K. Capsaicin receptor (TRPV1) and non-erosive reflux disease. Eur J Gastroenterol Hepatol 2006;18:263-270.

17. Matthews PJ, Aziz Q, Facer P, Davis JB, Thompson DG, Anand P. Increased capsaicin receptor TRPV1 nerve fibres in the inflamed human oesophagus. Eur J Gastroenterol Hepatol 2004;16:897-902.

18. Bredenoord AJ, Weusten BL, Curvers WL, Timmer R, Smout AJ. Determinants of perception of heartburn and regurgitation. Gut 2006; 55:313-318.

19. Woodland P, Al-Zinaty M, Yazaki E, Sifrim D. In vivo evaluation of acid-induced changes in oesophageal mucosa integrity and sensitivity in non-erosive reflux disease. Gut 2013;62:1256-1261.
20. Ugawa S, Ueda T, Ishida Y, Nishigaki M, Shibata Y, Shimada S. Amiloride-blockable acid-sensing ion channels are leading acid sensors expressed in human nociceptors. J Clin Invest 2002;110:1185-1190.

21. Farré R, Fornari F, Blondeau K, et al. Acid and weakly acidic solutions impair mucosal integrity of distal exposed and proximal non-exposed human oesophagus. Gut 2010;59:164-169.

22. Cicala M, Emerenziani S, Caviglia R, et al. Intra-oesophageal distribution and perception of acid reflux in patients with non-erosive gastrooesophageal reflux disease. Aliment Pharmacol Ther 2003;18:605-613.

23. Drewes AM, Schipper KP, Dimcevski G, et al. Gut pain and hyperalgesia induced by capsaicin: a human experimental model. Pain 2003;104:333-341.

24. Jones RC 3rd, Xu L, Gebhart GF. The mechanosensitivity of mouse colon afferent fibers and their sensitization by inflammatory mediators require transient receptor potential vanilloid 1 and acid-sensing ion channel 3. J Neurosci 2005;25:10981-10989.

25. Bulsiewicz WJ, Shaheen NJ, Hansen MB, Pruitt A, Orlando RC. Effect of amiloride on experimental acid-induced heartburn in non-erosive reflux disease. Dig Dis Sci 2013;58:1955-1959. 